Manuelle Medizin 2018 · 56:343-345

https://doi.org/10.1007/s00337-018-0448-8

(c) Springer Medizin Verlag GmbH, ein Teil von Springer Nature 2018

CrossMark

\section{Rigobert Klett}

Deutsche Gesellschaft für Manuelle Medizin (DGMM), Langgöns, Deutschland
Auf dem 121. Deutsche Ärztetag vom 08. bis 11. Mai 2018 wurden u. a. wichtige Beschlüsse zur Novellierung der MusterWeiterbildungsordnung gefasst, hierunter auch Titel, Definitionen und Mindestanforderungen (sog. Kopfteil) für die Zusatz-Weiterbildungen (ZWB). Für die ZWB „Manuelle Medizin“ beinhaltet die Beschlussfassung eine nichtakzeptable, qualitätseinschränkende Neuerung dahingehend, dass zukünftig nach Erlangung einer Facharztanerkennung in einem Gebiet der unmittelbaren Patientenversorgung die Kursweiterbildung über $320 \mathrm{~h}$ alternativ durch 12 Monate Weiterbildung unter Befugnis an dafür geeigneten Weiterbildungsstätten ersetzt werden kann. Im Vorfeld des Deutschen Ärztetags fand ein ausführlicher Meinungsaustausch zwischen Vertretern der Bundesärztekammer (B̈̈K) und der Deutschen Gesellschaft für Manuelle Medizin (DGMM) statt, wobei die DGMM vonseiten der BÄK über die von ihrer Ständigen Konferenz „Ärztliche Weiterbildung“ geplanten Änderung zur Ersetzbarkeit der Kursweiterbildung durch eine 12-monatige klinische Tätigkeit völlig im Unklaren gelassen wurde. Die Information erreichte die DGMM erst nach Abschluss der beratenden Gespräche mit der BÄK einige Tage vor dem Deutschen Ärztetag. Hierdurch waren die Reaktionsmöglichkeiten der DGMM im Vorfeld des Ärztetags massiv

Anhang: Stellungnahme der DGMM zur beabsichtigten alternativen Einführung einer einjährigen klinischen Weiterbildungszeit zur Erlangung der Zusatzweiterbildung "Manuelle Medizin", mit freundl. Genehmigung der Deutschen Gesellschaft für Manuelle Medizin (DGMM) e. V., @ alle Rechte vorbehalten. eingeschränkt. Trotz der Kürze der Zeit übersandte die DGMM vor dem Ärztetag noch eine Stellungnahme an die Ständige Konferenz „Ärztliche Weiterbildung" der BÄK zu der zu erwartenden Qualitätsverminderung und wohl nicht möglichen Umsetzbarkeit der Ausbildung im klinischen Alltag und der dadurch zu befürchtenden Reduktion der Patientensicherheit (Anhang). Ergänzend wurde mit Unterstützung von Prof. Johannes Buchmann über die Ärztekammer Mecklenburg-Vorpommern ein Beschlussantrag an den Ärztetag gestellt, die alternative Möglichkeit des Erwerbs der ZWB „Manuelle Medizin“ über 12 Monate klinische Weiterbildung wieder zu streichen. Dieser Antrag wurde allerdings nicht zur Diskussion auf dem Ärztetag zugelassen. Trotz aller kurzfristig noch möglichen Interventionsmöglichkeiten, die u.a. auch von der Deutschen Gesellschaft für Physikalische Medizin und Rehabilitation (DGPMR) und dem Berufsverband der Rehabilitationsärzte Deutschlands durch entsprechende Eingaben an den Präsidenten der BÄK und die Delegierten des Ärztetags unterstützt wurden, konnte die für die manuelle Medizin als äußerst negativ einzustufende Änderung zum Erwerb der ZWB nicht verhindert werden.

Allerdings ist von der negativen Entwicklung nicht nur die manuelle Medizin betroffen, zumindest teilweise ist auch bei den ZWB Ernährungsmedizin, Homöopathie, Krankenaushygiene, Naturheilverfahren, Palliativmedizin, Sexualmedizin und Sportmedizin ein Ersatz einer Kursweiterbildung durch klinische Tätigkeit möglich. Bei Durchsicht des
Beschlussprotokolls des 121. Deutschen Ärztetags zeigt sich wohl unter dem Eindruck des zunehmenden Ärztemangels eine gewisse Systematik hin zu einer Vereinfachung der Weiterbildung im Sinne von geringeren Kosten und geringerem Zeitaufwand für die weiterzubildenden Kollegen. So wurde beispielhaft auch eine für die Akupunktur beantragte Erhöhung der Ausbildungsstunden von 200 auf $300 \mathrm{~h}$ abgelehnt mit der Begründung zusätzlicher Kosten für den Arzt.

\section{\) Zukünftig kann die Kursweiterbildung über $320 \mathrm{~h}$ durch 12 Monate klinische Weiterbildung ersetzt werden}

Sicherlich besteht die Notwendigkeit, dem Ärztemangel entgegenzuwirken, allerdings muss hier wie im Fall der ZWB „Manuelle Medizin“ die Sinnhaftigkeit einer Ausbildungsvereinfachung auf Kosten der Qualität und der Patientensicherheit infrage gestellt werden. Als irritierend stellt sich zudem die Tatsache dar, dass insbesondere ZWB von der Vereinfachung betroffen sind, die einen großen Anteil der „sprechenden Medizin“ beinhalten. Gerade die „sprechende Medizin“ wird in einem aktuellen Referentenentwurf des Ministeriums für Gesundheit zu einem Gesetz für schnellere Termine und bessere Versorgung als wesentlich angesehen und soll durch eine bessere Vergütung gestärkt werden.

Für die manuelle Medizin ist es von entscheidender Bedeutung, dem drohenden Qualitätsverlust und der drohenden Reduktion der Patientensicherheit ent- 
gegenzuwirken und die in den letzten Jahren diesbezüglich erzielten Errungenschaften zu bewahren und weiterzuentwickeln. Berufspolitisch bleibt hier aktuell primär nur die Möglichkeit, auf Ebene der Landesärztekammern die Umsetzung der Muster-Weiterbildungsordnung im Sinne der manuellen Medizin zu begleiten und auf entsprechend qualitativ hohe Anforderungen an die Erteilung einer Weiterbildungsbefugnis hin $\mathrm{zu}$ arbeiten. Dies gelingt umso besser, desto umfassender die Inhalte der manuellen Medizin wissenschaftlich untermauert sind.

Ich möchte daher alle Mitglieder der DGMM und Unterstützer der manuellen Medizin aufrufen, sich an der wissenschaftlichen Weiterentwicklung der manuellen Medizin zu beteiligen und, wenn möglich, ihren persönlichen Einfluss in den Landesärztekammern auf die entsprechenden Entscheidungsgremien geltend zu machen. Nur so kann zum Nutzen der Patienten das bisher Erreichte für die manuelle Medizin erhalten und ausgebaut werden.

Mit freundlichen Grüßen, Rigobert Klett

\section{Korrespondenzadresse}

\section{Prof. Dr. Rigobert Klett}

Deutsche Gesellschaft für Manuelle Medizin (DGMM)

Fichtenweg 17, 35428 Langgöns, Deutschland rigobert.klett@radiol.med.uni-giessen.de

Interessenkonflikt. R. Klett weist auf folgende Beziehungen hin: EristPräsident der Deutschen Gesellschaft für Manuelle Medizin sowie 2. Vorsitzender des Dr. KarlSell-Ärzte-Seminars Isny-Neutrauchburg (MWE).

\section{Anhang}

Stellungnahme ${ }^{1}$ der DGMM

zur beabsichtigten alternativen

Einführung einer einjährigen

klinischen Weiterbildungszeit

zur Erlangung der Zusatz-

weiterbildung "Manuelle

Medizin"

Die Deutsche Gesellschaft für Manuelle Medizin (DGMM) hat die Bestrebungen der Ständigen Konferenz „Ärztliche Weiterbildung " zur Kenntnis genommen, die Weiterbildung "Manuelle Medizin“ neben dem bisher im Kurssystem möglichen Erwerb auch innerhalb eines Jahres Vollzeit-Weiterbildung möglich zu machen. Die DGMM wendet sich entschieden gegen die Installation dieser Möglichkeit aus folgenden Gründen:

Noch vor 30 Jahren galt die Impulsmanipulation an der Wirbelsäule als risikobehaftet mit z. T. schweren Komplikationen und dramatischen Verläufen. Durch die kontinuierliche intensive Arbeit der wissenschaftlichen Gruppierungen innerhalb der DGMM, namentlich der drei deutschen wissenschaftlichen Seminare für manuelle Medizin (Ärztevereinigung für Manuelle Medizin [ÄMM], Deutsche Gesellschaft für Muskuloskeletale Medizin [DGMSM], Gesellschaft der Ärzte für Manuelle Wirbelsäulenund Extremitätentherapie [MWE]), ist es gelungen, diesen Sachverhalt ganz entschieden zu ändern. Durch die konsequente Einführung und Durchsetzung der DGMM-Regeln zur „sanften Manipulation" konnte dieses Verfahren der Wirbelsäulenbehandlung sicher gemacht werden, und es konnte auch innerhalb des Kurssystems effizient und suffizient an alle Bewerber um die Zusatz-Weiterbildung (ZWB) vermittelt werden. Dazu war eine systematische Analyse der biomechanischen Zusammenhänge, eine umfassende Einordnung der Verfahren im Sinne der translationalen Forschung und der klinischen Versorgungsrealität sowie ein intensives Training der Bewerber um die ZWB erforderlich. Be-

\footnotetext{
1 Stellungnahme der Deutschen Gesellschaft für Manuelle Medizin (DGMM) e.V., @ DGMM, alle Rechte vorbehalten.
}

gleitend war intensive und systematische Schulung aller Instruktoren innerhalb der Kurse für manuelle Medizin unabdingbare Voraussetzung. Es ist auch in den letzten 30 Jahren auf diesem Wege gelungen, die Komplikationsrate für die Therapieverfahren der manuellen Medizin bei Beachtung aller Vorsichtsmaßnahmen gegen NULL zu bringen. Auch aus neurologischen Positionierungen wird diese Auffassung der DGMM geteilt. Heute ist die manuelle Medizin unwidersprochen als evidenzbasierter wirksamer Bestandteil aller Therapiestrategien an der Wirbelsäule anerkannt. Dies schlägt sich nieder in den Leitlinien zum nichtspezifischen Kreuzschmerz, spezifischen Kreuzschmerz, Thoraxschmerz, zur axialen Spondylarthritis und jüngst auch in der neurologischen Leitlinie zum radikulären Armschmerz.

Die alternative Verlagerung der Weiterbildung zur ZWB „Manuelle Medizin“ in den klinischen Alltag hätte zur Folge, dass mit sehr großer Wahrscheinlichkeit wichtige Standards in Theorie und Praxis eingebüßt würden und dass die erreichten Erfolge für die manuelle Medizin in Gefahr geraten würden. Es ist immerhin eine effiziente, kostengünstige und heute für den korrekt Weitergebildeten praktisch risikofreie Therapiemethode mit hoher Compliance, in die auch sehr viele der wissenschaftlich belegten osteopathischen Verfahren Eingang gefunden haben. Die 320 Unterrichtseinheiten, die in allen bundesdeutschen Ärztekammern in präzise formulierten Curricula einschließlich der Stundeneinteilung und in jüngster Vergangenheit auch nach den Kriterien der Miller-Pyramide niedergelegt sind, bieten eine sichere Voraussetzung für den Erwerb der ZWB und Anwendung der genannten Verfahren.

Das Argument, dass heranwachsende junge Fachärzte sehr viele Fertigkeiten im Kurssystem mit entsprechendem finanziellem und urlaubstechnischem Aufwand $\mathrm{zu}$ realisieren haben, ist bestimmt richtig. Die Kurse für manuelle Medizin siedeln sich jedoch im absolut untersten Preissegment verglichen mit allen anderen Weiterbildungen an und sind heute von der Organisation her so strukturiert, dass im Durchschnitt an Wochenenden 
über $1 \frac{1}{2}$ bis 2 Jahre diese ZWB solide und gut fundiert erworben werden kann.

Im klinischen Alltag würde die Umsetzung bedeuten, dass der Weiterbildungsbefugte, um innerhalb eines Jahres den Rahmen von 320 curriculären Stunden Weiterbildung $\mathrm{zu}$ erfüllen, mindestens an 65 Tagen pro Jahr bis zu 5 h Frontal- und Gruppenunterricht sowie praktische Unterweisung und Supervision des Übens der Kolleginnen und Kollegen aneinander durchführen müsste, was aus der Sicht der DGMM derzeit in keiner bestehenden Form klinischer Institutionen - weder operativ noch konservativ realistisch darstellbar ist. Der Erwerb der Fertigkeiten im Rahmen der ZWB „Manuelle Medizin“ ist insbesondere durch Üben in den Kursen, üblicherweise durch gegenseitiges Üben von Kolleginnen und Kollegen aneinander, realisiert und ist allein durch theoretischen Unterricht oder durch Üben an Patienten nicht darstellbar. Zumindest am Beginn der Ausbildung bestünde beim direkten Üben am Patienten ein deutlich erhöhtes Risiko.

Seitens der DGMM werden also, insbesondere im Sinne der Qualitätserhaltung und der Sicherheit der Patienten, ganz erhebliche Bedenken gegen diesen Plan der Realisation innerhalb einer klinischen Weiterbildung erhoben und die entsprechenden Strukturen und Gruppierungen aufgefordert, dieses Verfahren noch einmal zu überdenken. Es ist nicht nachzuvollziehen, warum weiterhin viele andere Kurse zum Erwerb einer ZWB außerhalb der klinischen Ausbildung eingeordnet werden, während eines der sensibelsten Fächer mit besonders hohen Anforderungen an das motorische Lernen jetzt plötzlich im klinisch operativen oder klinisch konservativen Betrieb erwerbbar werden soll.

Die täglichen Arbeitspläne der Ärzte sind, wenn man den Veröffentlichungen und den Verlautbarungen vertrauen möchte, ohnehin so gestrickt, dass für Aufgaben über die klinische Arbeit und die Dokumentation hinaus kein Raum bleibt. Unbenommen aller Argumente ist die Vermeidung einer Gefährdung der Patientensicherheit durch unzureichende Weiterbildung im klinischen Alltag vordringlich. Die Ärztinnen und Ärzte, die im klinischen Bereich die Verant- wortung für die Weiterbildung zur ZWB „Manuelle Medizin“ übernehmen müssten, müssten auf jeden Fall den Standard der Qualifikation von Instruktoren in den DGMM-Kursen zur ZWB „Manuelle Medizin“ erfüllen und müssten durch die Landesärztekammern qualitätskontrolliert werden. Hier wird intensiv davor gewarnt, mühsam erreichte Qualitätsstandards zur Patientensicherheit und zur wissenschaftlichen Untermauerung manualmedizinischer Verfahren jetzt zu gefährden.

\section{Selbsthilfebuch wirkt gegen Burnout und Stress}

Erstmals haben Forscher die Wirksamkeit eines Selbsthilfebuches, das auf der Akzeptanz- und Commitmenttherapie (ACT) beruht, untersucht. Dieses hat das Potenzial, ganz ohne Therapeutenkontakt Burnout, Stress und Symptome der Depression zu reduzieren.

Stress am Arbeitsplatz stellt eine Bedrohung für die psychische und körperliche Gesundheit dar und ist mit Einschränkungen und hohen sozioökonomischen Kosten verbunden. Selbsthilfebücher könnten eine günstige Alternative zu Programmen bieten, die von Experten und Therapeuten geführt werden. Das Selbsthilfebuch zeigt auf, wie Burnout mit emotionaler Rigidität zusammenhängt und beschreibt wie ACT diese innere Rigidität verändern kann. Durch Achtsamkeit und Akzeptanz soll ein flexiblerer Umgang mit schwierigen Gedanken und Emotionen ermöglicht werden.

Für die Studie wurden Personen aus unterschiedlichen Berufsgruppen mit moderaten und schweren Stresswerten zufällig einer von zwei Gruppen zugewiesen. Die eine Gruppe arbeitete das Selbsthilfebuch sofort während fünf Wochen durch. Die zweite Gruppe erhielt das Buch erst nach einer Wartezeit.

Personen, welche das Selbsthilfebuch erhielten, zeigten eine deutlich stärkere Verbesserung in den Bereichen Stress, Burnout, Depressionssymptome und Wohlbefinden als Personen in der Wartelistegruppe. Drei Monate nach Abschluss berichteten die Studienteilnehmer von einer weiteren signifikanten Abnahme von Stress, Burnout und Depressionssymptomen.

Aus Sicht der Studienleiters ist ein Selbsthilfebuch ohne Therapeutenkontakt jedoch nicht für alle Personen geeignet sondern könnte ein erster Schritt im Sinne eines gestuften Versorgungskonzeptes sein.

Literatur: P. Hofer, M. Waadt, R. Aschwanden (2017) Work \& Stress, doi: $10.1080 / 02678373.2017 .1402389$

Quelle: Universität Basel, www.unibas.ch 